\title{
A PROPÓSITO DE LAS RESTRICCIONES DE ORDEN EN LAS SECUENCIAS DE CLÍTICOS EN ESPAÑOL
}

\section{CONSIDERACIONES PREVIAS.}

I.I. Is un hecho bien conocido que el español impone ciertas restricciones a las secuencias de clíticos. En este trabajo estudiaremos solamente las cuestiones relativas al orden "interno" de tales secucncias, dejando aparte el problema de su colocación en la oración. Is decir, desde este punto de vista, las oraciones

$$
\left[\begin{array}{l}
\text { se lo quiero decir } \\
\text { quiero decírselo } \\
\text { "quiero se lo decir }
\end{array}\right] \text { vs. }\left[\begin{array}{l}
\text { *lo se dice } \\
* \text { digalose } \\
* \text { quicro lo se decir }
\end{array}\right]
$$

reflejan un único hecho: la posibilidad, en español, de la secuencia de clíticus SE LO y la agramaticalidad de LO SE. En lo sucesivo "orden de una sccuencia de clíticos» se utilizará en este sentido restringido.

Muclias de las gramáticas del español indican explícitamente que los clíticos castellanos han de aparecer en secuencias que respeten el orden SLi-2. ${ }^{a}$ persona-I. ${ }^{a}$ persona-3. ${ }^{a}$ persona (de aquí en adelante, SE II I III), como por ejemplo Bello (p. 305), Gili Gaya (p. I67). En cl capítulo fundamental de su Tesis de Doctorado, D. Perlmutter lia intentado demostrar que tal orden, básicamente correcto, no puede ser producido por una gramática transformacional del español más que si aplicamos un conjunto de restricciones al educto del componente transformacional de esa gramática: una especie de filtro que retendría a las sccuencias que no se ajustaran al orden que establece. (Traduzco output/input por 'educto/aducto' según la sugerencia, creo que bien fundada, de C. P. Otero.) 
La tesis de Perlmutter, que podría parecer un bizantinismo que nada agrega a lo ya sabido anteriormente, tiene una importancia teórica grande. Ein la Teoría "standard" generativa (la de Aspects...) sólo son verdaderas cstructuras profundas las proyectadas por el componente transformacional en estructuras de superficie gramaticales. Es, por tanto, únicamente el componente transformacional el que actúa como filtro: una estructura profunda que provoque el bloqueo de alguna T. obligatoria, no llegará como aducto al componente fonológico, y por cllo no scrá una verdadera estructura profunda.

Este encuadre teórico es incompatible con las conclusiones de Perlmutter en dos aspectos interdependientes: en primer lugar, al nostrar que existen en español uoraciones agramaticales que no pueden ser caracterizadas como tales por el medio habitual, a saber: el bloqueo de las transfornaciones obligatorias... se hace necesario reforzar la teoria gramatical añadiéndole restricciones de la estructura superficial (surface structurc constraints) o condiciones eductivins (output conditions)" - Perlmutter, I968, p. I9-, restricciones que debe satisfacer toda estructura profunda proyectada por las reglas transformacionales. En segundo lugar, y cono consecuencia de lo anterior, debemos concluir que hay estructuras profundas bien construidas -i. e. que no provocan el bloqueo de ninguna $T$. obligatoria - que, sin embargo, subyacen a estructuras superficiales agramaticales. Con ello ponemos en cuestión la noción básica de la teoría transfornacional, considerada asi explícitamente por Cliomsky (Aspects, p. I3I).

r.2. Iin estc trabajo intentaremos razonar un enfoque completamente diferente del problena, tanto a nivel de adecuación empírica (único en el que se mueve la gramática tradicional), como en el de la adecuación cxplicativa de la Teoria Lingüistica General (que es el verdadcramente importanite para Perlinutter). Trato, en primer lugar, de mostrar que el filtro propuesto hasta ahora para seleccionar las sccuencias de clíticos gramaticales y eliminar las que no lo son, tiene un cedazo demasiado grosero; es decir, todas las secuencias que retiene son agramaticales, pero no todas las que deja pasar son gramaticales. In segundo lugar, propongo un conjunto más completo de condiciones de gramaticalidad que subsane el $\mathrm{mal}$ funcionamiento antes señalado. Discutimos luego las implicaciones que todo ello proyecta sobre la teorfa general. El cuarto -y más importante- punto se reficre a la formulación de dicho conjunto de condiciones, se discute su ubicación como condiciones eductivas y se llega a la conclusión de que, en realidad, no forman un tal conjunto sino que pertenecen a categorías de res- 
tricciones muy diferentes, pero en iningún caso situadas en la cstructura superficial; las restricciones sobre el orden quedan, además, nítidamente destacadas sobre las demás.

I.3. Para la argumentación utilizaremos una modalidad de español que 110 admite leísmos, laísmos, ni, por supuesto, loísmos. Es Ia utilizada mayoritariamente en Andalucia, Extremadura, Noroeste peninsular y América hispana. De cualquier forma, esta elección no ticne influencia alguna sobre las conclusiones finales: las restricciones sobre cl orden de clíticos son válidas para todo dialecto español culto. Iiste extremo quedará claro en la última parte de este trabajo.

2. IiN BUSCA DE LAS CONDICIONLS NECLSARIAS Y SUFICILNTES DE: GRAMATITCALIDAD.

2.I. Supongamos como regla de ordenación de las secuencias de cliticus la siguiente:

(I) SIE II I III.

Ia regla, que ya dijimos que es bien conocida, hay" que interpretarla con una scrie de convenciones muy restrictivas (que Perlmutter discute, mientras que las gramáticas tradicionales las dan por supuestas): a) La regla marca una relación de orden obligatorio, pero no que cada una de liss posiciones ordenadas se tenga (fue ullenar" obligatoriamente: la secuencia SIE III es conforme a la regla. b) La relación de orden cutre las posiciones es estricta, esto es: transitiva, antisinétrica y noreflexiva. (Recordemos: sean $x, y, z$, posiciones de la regla (I). Una rclación de orden estricto entre ellas, por ejemplo "precede a" -que llamaremos $\mathrm{R}-$, impone:

- $n o$ cs cierto que $x \operatorname{Rx}$ (No es cierto que $x$ "precede a" $x$ )

- si $x R y$ entonces $x R z$

- si $\underset{y R y}{y R}$ entonces $\mathrm{x} \equiv \mathrm{y}(\mathrm{x}$ es idéntico a $\mathrm{y})$.)

Por cllo, la regla además de marcar un orden implica condiciones cuantitativas; por cjemplo, no se perinite más de un miembro de cada posición. I a secuencia SE III III no es gramatical por tanto. c) SE se enticnde como realización fonológica o cuasi-fonológica, ya que de otro dnodo la regla sería contradictoria pucs, naturalmente, en III queda 
englobado todo SE. d) La regla (I) ha de ser aplicada después de la regla que origina el "falso SE», que Perlmutter formula así:
(2) $\left[\begin{array}{l}\text { Pro } \\ \text { III } \\ \text { Dat. }\end{array}\right]$
$\left[\begin{array}{c}\text { Pro } \\ \text { III } \\ \text { Ac. }\end{array}\right]$

Il SE así originado debe sufrir posteriormente la restricción (I).

Las convenciones de interpretación de (I) que acabamos de enumerar nos indican que, en realidad, ésta no es una verdadera regla que reordene las secuencias üe clíticos, sino más bien una especie de filtro que deja pasar las secuencias que se ajustan al orden prescrito y que retiene a las demás, La operación de tamizado hay que entenclerla con un doble valor: por un lado, las secuencias retenidas quedan califiçadas como agramaticales; por otro, las secuencias que traspasan el filtro se considcran gramaticales. (I) es algo más que un conjunto de condiciones de gramaticalidad necesarias para toda secuencia; es (pretende ser) el conjunto de condiciones necesarias y suficientes para que una secuencia de clíticos sea gramatical. Fin este sentido, podrános đecir que, más que "un» filtro, se pretende que sea "el» filtro. Ello va implícito en las gramáticas tradicionales, que no dan ninguna otra condición de gramaticalidad; Perlmutter lo reconoce explícitamente en varios pasajes: "la condición eductiva (es) como un tamiz que determina las secuencias gramaticales de pronombres clíticos y descarta como agramaticales las secuencias que no se conforman a élı (p. I05 de la versión francesa). Naturalmente, si se descubren nuevas restricciones labrán de ser obligatoriamente añadidas a (I), para que éste siga manteniendo su carácter de "conjunto de condiciones suficientes», además de "necesarias".

2.2. Dos tipos de contraejemplos se pueden presentar en contra de (I). El primero sería el constituido por secuencias gramaticales que hayan sido eliminadas por la regla; no conozco caso alguno de este tipo, y por ello admitimos que (I) está integrado por condiciones necesarias. El otro tipo de contraejemplo serían las secuencias conformes a (I) y que, sin embargo, forman parte de oraciones agramaticales. Antes de pasar a discutirlo, tendremos que aclarar algunos puntos.

2.2.I. En una oración agramatical y que contiene clíticos hay que distinguir cuidadosamente las causas de tal agramaticalidad. Ora- 
ciones incorrectas y que, sin embargo, no son eliminadas por (I), pueden deberse a violación de reglas no relativas en absoluto a los clíticos (a su orden). Un ejemplo evidente sería «*se lo va»: la oración no es gramatical - como cn cambio lo es use lo cogen-, pero la conclusión correcta es que (in no admite un clítico acusativo; la agramaticalidad provienc de no tener en cuenta uno de los rasgos (¿sintácticos o semánticos?) del verbo. Otto ejemplo sería "*él se te me lo lee» (secuencia perfectamente ajustada a (I): juzgarlo o no gramatical dependerá siempre de que definamos previamente qué número y qué tipo de complementos admite «leet».

Hemos de distinguir, por tanto, entre gramaticalidad de la secuencia de clíticos y gramaticaliclad de la oración; el tema que nos ocupa es cl primero, $y$ es importante plantearse las condiciones bajo las cuales debemos atribuirle 1o segundo. Como principio general para accintar un presunto contracjemplo de (I), al ser agramatical y al mismo tiemuo acorde con tall regla, debemos presentar un caso "paralelo" y gramatical. Por "paralclo" cntendemos otro cjemplo con igual verbo (sin tener en cucintat número ni persona) y complementos. P'or tanto "cil sc te me lo leen. no puede considerarse como prueba en contra de (I), ya que no podemos aportar ejemplos paralelos en el sentido que hemos clado a este término; no hay oración de esquema «SE II I III + leer» que sea gramatical. Igual ocurre con "se lo va»: no existe ejemplo alguno gramatical del tipo "II I + in», flexionemos como flexionemos el vesbo.

2.2.2. El segundo punto que requicre aclaración se refiere a lo que Perlnutter llama "restricciones no-globales" (pp. 60 y ss.), término que no definc con precisión. Existen, desde luego, ejcmplos que, ajustándose a (I), no son gramaticales; la agramąticalidad, además, se debe a la secuencia de clíticos. Estos ejemplos constituirán, más que una jrucba en contra de la regla, testimonio de que ésta no es completa, no es el conjunto de condiciones de gramaticalidad suficiente. Por tanto, una granática adecuada del español debe incluir restricciones adicionales que eliminen tales ejemplos; estas restricciones, que llamamos no-globales, no son necesarias para toda secuencia, pero debemos añadirlas al conjunto de condiciones necesarias para transformarlo en el conjunto de condiciones necesario y suficiente. Las restricciones noglobales no son más que condiciones solamente necesarias para un cierto tipo de secuencia de clíticos. No conozco ningún estudio correcto de cllas, ni siquiera planteamiento alguno claro de su existencia. Perlmutter, que sólo las menciona en térninos vagos, las cntiende en la práctica más como restricciones que sufre cicrto tipo de verbos que 
como condicionantes del orden de los clíticos (p. ej., en la restricción (I40) de la p. I62). Ello equivale a afirmar la imposibilidad de formular condiciones suficientes que garanticen la gramaticalidad del orden de los clíticos en términos de las caracteristicas de los mismos clíticos solamente: habría que recurrir además a caracteristicas de verbos.

In los próximos parágrafos vercmos que ello no es asi, y que es pcsible formular las restricciones no-globales utilizando simplemente las características de los clíticos.

2.3.I. - El caso ME LE/TE LE.

La oración

(3) Yo me le acerco NIE $\rightarrow$. LE

es perfectamente posible para un hablante no leista, y se opone precisamente a

(4) Yo me lo acerco $\mathrm{ME} \leftarrow$ LO

Iin (3) soy yo el que me mucvo hacia lo otro; en (4) muevo a lo otro hacia mí (es lo que quercmos indicar con las flechas). Obsérvese que para un liablante leista (3) scría ambiguo.

Lo curioso es que la oración

(5) *Yo te le acerco TE $\rightarrow$ IE es agramatical, y si queremos expresar tal idea, habremos de recurrir a los pronombres tónicos

(G) Io te acerco a (hacia) él TE $\rightarrow$ EL Iixactannente igual ocurre con

(7) :Tú me le acercas ME $\rightarrow$ LE frente a

(8) Tú me lo acercas

(9) Tí te le acercas

$\mathrm{MIE} \leftarrow \mathrm{LO}$

$\mathrm{TE} \rightarrow \mathrm{IE}$

Iis decir, (5) y (7) no son gramaticales, pese a amoldarse a (I). Ejemplos del mismo tipo podemos encontrarlos fácilmente con verbos como arrimarse (yo me lo arrimo vs. yo me le arrimo, pero *tú me le arrimas), presentarse, rendirse, entregarse, apartarse, subirse, arrojarse...; un ejemplo de Rómulo Gallegos (citado por Holton, Ig6o): "¿Qué vas a ganar con incorporártele a esa gente?\#; otros de Miró, tomados de Fernández Ranúrez: "Su cuñada se les apartó», "Iilla se le precipitó con una sonrisa»...

Para buscar una explicación a todo esto hay que salirse del marco de (I) y tener en cuenta otras características de los clíticos. Intre todos los ejemplos antcriores, los correctos obedecen a los tipos de secuencia

Reflexiro-Dativo: (3) y (9) A partir de ahora escribiremos Dativo-Acusativo: (4) y (S) RI', D'T, AC. 
Si tras (I) imponemos la restricción global (con las mismas convenciones interpretativas)

(I0) RF-DT-AC

queda explicada la gramaticalidad de (3), (9), (4) y (S), y la agramaticalidad de (5) y (7), que cumplen (I), pero violan la regla (I0), ya que sigucn el orden $\mathrm{AC}-\mathrm{D}$ 'T.

(Unicamente en Heger (I967) he visto que se afirmara como correcta una construcción del tipo de (5) y (7) en una modalidad de español hablado en Anérica. En ningún otro sitio he encontrado confirmada tan extraña construcción, tan repugnante a la conciencia lingüística del hablante peninsular que hace falta explicar mediante flechas lo que de otra manera se entendería como leísmo. La poca exactitud de muchas de las citas de dicho artículo -el calificar de leísta el cspanol peninsular, por ejemplo- induce a pensar que nos hallamos ante otra afirmación un tanto atrevida).

\subsubsection{La importancia del falso $S E$}

A primera vista (I0) podría tomarse como otro conjunto de condiciones necesarias globales; oraciones gramaticales como use te lo come» "te me lo llevas", "se lo lava" (en los dos sentidos: RF-AC y DT-AC). son permitidas tanto por (I) como por (IO). Y como además este último cs un conjunto de condiciones más estricto, ya que elimina correctamente oraciones como (5) y (7) que (I) deja pasar, podríamos pensar que, simplemente, las condiciones de ( $\mathrm{r}$ ) no son sino parte de las de (IO): bastaria cutonces con establecer esta última regla.

Que la anterior suposición no es descabellada, lo demuestra el que M. E. Moen tenga que criticarla todavía en su artículo de I966: "La leyenda de que los pronombres reflexivos preceden a los dativos, y éstos a lus de acusativo, ha pasado entre los autores americanos de textcs (de español), de gencración en generación” (p. 9). Y sin embargo es muy fácil de demostrar que no todas las condiciones incluídas en ( $I$ ) forman parte de (Io): en la oración "yo me le como el bocadillo (a Juan)" podríamos intentar pronominalizar el complemento directo, lo que daría

(II) *Yo me le lo como

$y$, con la aplicación obligatoria de la regla del falso SE:

(I2) *Yo me se lo como

oración que es eliminada correctamente por (I) pero no por (IO), ya que su orden es RF-DT-AC, mientras no especifiquemos más lo que entendemos por RF. Queda evidenciado que el conjunto de condiciones necesarias representado por (I) no es un subconjunto propio del re- 
presentado por (Io), y que es necesario formular ambas reglas para tratar de alcanzar el conjunto de condiciones necesario y suficiente.

\subsubsection{El caso II I-}

Pero octure, adcmás, cue henos ido demasiado deprisa al dar por sentado que (ro) expresaba un conjunto de restricciones globales, necesarias para toda secuencia de clíticos gramatical. En

(I3) Yo te me acerco TE $\leftarrow$ MEE

tenemos el orden DT-RF que infringe (ro) pero que es gramatical: (ro) no es, por tanto, una restricción globalmente necesaria. Al añadirla a (I) hay que hacerlo con salvedades, ya que si no eliminamos secuencias gramaticales al imponer demasiadas condiciones. En general, es la secuencia II I- la que no sigue la regla (Io):

(I4) Nosotros os nos humillamos DT - RF

(I5) ¿Adónde os me llevan? (Lazarillo de T.) $\Lambda \mathrm{C}-\mathrm{DT}$ (l:jejuplus ambors que he tomado de $A$. Alonso \& Henriquez Ureña, 1. 94).

Habria que elaborar entonces una especie de filtro doble: el primero scria (I), y todo lo que lo traspasara, menos II I-, debería sufrir además el segundo filtro, o sea (ro). El educto final serían las secuencias gramaticales; las secuencias retenidas serian agramaticales:

(I6)

a)

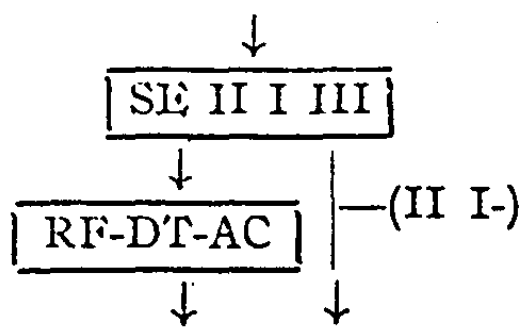

Secuencias gramaticales

(Mr. I. Moen, que propugna (r) como único filtro y rechaza (ro), reconoce que oraciones como

(I7) *No te me voy no son gramaticales para gran parte de los hablantes de español, en contra de

(I8) No te me vas que sí lo es. Para completar entonces (I) —que dejaria pasar a la agramatical (I ) - propone la siguiente regla:

(I9) II-I-verbo en I. a persona $\rightarrow$ I-verbo en $I .{ }^{a}$ persona-lccución preposicional (a ti, de ti, etc.). 
(I7) se convertiría cutonces en "no me voy de tin (?) o algo semejante. Lil problema, sin embargo, es que las oraciones de tipo (5) y (7) sigucn pasando la combinación de filtros (I)-(Ig), siendo sin embargo incorrectas, lo que demuestra la necesiclad de (xo). Por otra parte, el caso de la sucesión II I- no es tan sencillo, como lo denuestran las uracioncs gramaticales "te me presento", "te me rindo"... que (Ig) clinina, y que alıora pasaremos a discutir.)

\subsubsection{Posibles refinamientos de (Iб).}

(16) es un sistema de restricciones que funciona bien para una variedad de español que admite tanto oraciones del tipo (I3)

(13) Yo te me acerco como del tipo

(I7) 'Te me voy

modalidad que se habla, por ejemplo, en Galicia por influencia de la lengua vernácula: el gallego posee un clítico (que Carballo Caleto (p. 22.3) llama "dativo de solidaridad", y que hace referencia siempre al interlocutor; es una manera de englobarlo en lo que se dice, de dar por supucsto que a él le interesa: un gallego, haciendo un calco sintáctico, puede decir "te hace muy buen tiempo", "yo le vengo siendo de Mos" (C. J. Cela). Por lo mismo, es perfectamente admisible en este dialecto una oración como "él se te me lo comió» (equivalente a "él se me lo comió, ¿sabes?" o algo parecido), que aprovecha al máximo las posibilidades del filtro (I 6 ).

Alora bien, la variedad más extendida del español -el oficial, desde luego- no admite ( $\mathrm{I} 7$ ), y habría que restringir los casos de II Ique no pasan por el subfiltro ( $\mathrm{r} 6 \mathrm{~b}$ ), haciendo que los del tipo ite me voy" sí lo hicieran -y que, por tanto, sean eliminados, ya que su orden es D'I-Kir- (Obsérvese que (I7) es un verdadero contracjemplo de la regla (IG), ya que una oración como "te me vas", paralela a (I7) según nuestra terminología, es perfectamente correcta para toda variedad del español.)

Pese a las apariencias, el problema no es complicado, pero exige desarrollar someramente una teoría de las pronominalizaciones, cosa que no hemos hecho hasta alora. Teoría que, por otra parte, es uno de los tópicos más debatidos actualmente en el marco de la gramática generativa.

En toda teoría de la pronominalización juega un papel fundamental el mecanismo que asigna a los pronombres una determinada correferencia con sintagmas nominales de la oración. Este mecanismo puede enten- 
derse trasformacionalmente (por ejemplo, Bach (1968); McCawley (r970) y en general toda la semántica generativa), o como parte del componente semántico, tal como hace la "semántica interpretativa", de la que puede ser Jackendoff (1972) (cap. 4) su más claro exponente. Nos interesa scñalar aquí, dejando a un lado esta discusión, la pertinencia. para nuestro problema de una clasificación de los clíticos que parta de la interrelación entre la estructura funcional de la oración y su estructura. de correferencia. Aceptando unas nociones funcionales de sujeto y complemento directo que sigan en sus grandes líneas las de Chomsky (1965) (Aspects), tendriamos tres tipos de clíticos:

- Correferenciales con el sintagma nominal sujeto (a partir deahora denominados $(\mathrm{R})$.

- Correferenciales con el complemento directo (lo que hemos denominado $\mathrm{AC}$ ).

- Correferenciales con otros tipos de complemento (serían los DT).

Hay que resaltar varias características de esta clasificación. La primera es que las tres características de clíticos no son mutuamente excluyentes (aunque si lo son, por definición, AC y DT): Un CR puede ser correferencial con el sujeto ("me le como el bocadillo"), con el sujeto. y el complemento directo ("me lavou), y con el sujeto y otro tipo de complemento ("me lavo la cara»). Obsérvese que los $C R$ también correferenciales con un complemento son los que, más o menos, conocemos. por "reflexivos». (Queda asi reflejada la división tradicional de los reflexivos ell acusativos y dativos; cf. Academia, § 249). Podríamos esquematizar todo esto así:

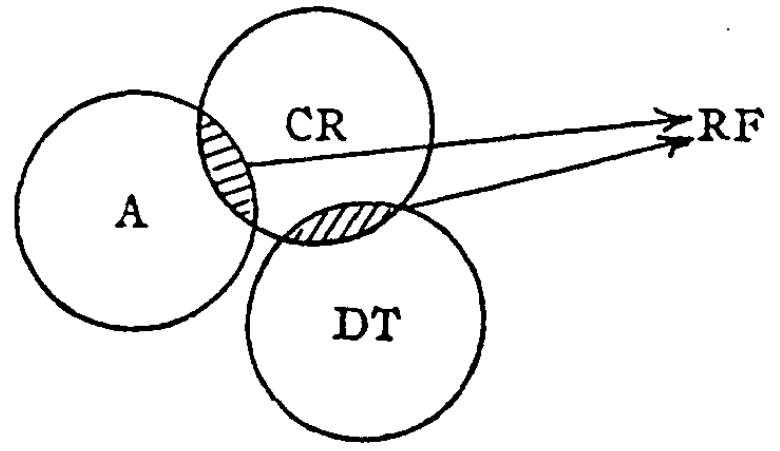

Io segundo es que evitamos cualquier mención de la noción, tan poco clara, de complemento indirecto, al definir a los clíticos DT negativamente en relación con los $\mathrm{AC}$.

Lo tercero es que, a efectos de las reglas de ordenación, la categoría $C R$ cs "dominante»: cuando un clítico es $C R$ y al mismo tiempo acusativo o dativo, se le considera primordialmente $\mathrm{CR}$; es decir, la correferencia con el sujeto domina a todas las demás. Este hecho se refleja incluso. 
morfológicamente: en tercera persona, el clítico CR es SE, ya sea dativo, acusativo o simplemente $C R$. En gallego ocurre algo parecido con el clitico de segunda persona, que distingue $A C$ (tc) de $D^{\prime} T$ (che), pero que se unifica cu "te» cuando es $C R$, sin tener cn cuenta si es además D'T - AC. (Cfr. Carballo Calero, p. 22 I y ss.).

Lin cuarto lugar hay que notar que al transformar

(Io) RIF-DT-AC

en

(I8) CR-DT-AC

propugnamos una generalización de gran importancia: no sólo los $R F$ sino todo correferencial con el sujeto ha de colocarse en primer lugar; ello explicaría el que en "tú te le comes el bocadillo" aparezca el orden II III, mientras que en "él se te come el bocadillo" sea III II (donde. ni "te" ni "se" son verdaderos reflexivos). Lo que en un principio puede aparecer como idiosincrasia del SE (tanto el reflexivo como los llamados. tradicionalmente (impersonal", "pasivo-reflejo», "ćtico"..., etc.) no sería sino la manifestación externa de una regularidad profunda: el asignar el primer lugar, en exclusiva, a los CR.

El considerar, por otra parte, al SE impersonal y pasivo-reflejo como correferenciales de un sujeto profundo, es algo en que han coincidido lingüistas de las más diversas tendencias. Oca (IgI4) titula su artículo sobre el SE impersonal, muy significativamente, "El pronombre SE en nominativol: no sería tan sólo correferencial con el sujeto sino, más bien, el sujeto. $\mathrm{Y}$ casi sesenta años después, y dentro de las últimas tendencias generativas, Schroten (I972) dedica un sugestivo trabajo a demostrar que hay una coincidencia básica (profunda) en todos los tipos (superficiales) de SE: provendrían siempre de la aplicación de la "transformación reflexiva», al encontrarse un sintagma nominal idéntico como Agente y como Complemento. Heles Contreras (I973, nota I) está trabajando actualmente en el mismo sentido ("Creo cn cstos momentos que SE -en "se alquilan apartamentos", por ejemplo- sc introduce por Reflexivizacióny), y anuncia una monografía drdicada al tema. (Como consecuencia de todo lo dicho hasta aquí se puede afirmar que consideramos $C R$ a todo clítico que coincida en persona con el sujeto, con la salvedad de que en la tercera persona existe la forma especializada SE, y ninguna otra puede ser CR. Por supuesto, cl falso SE ha de ser considerado DT.)

Ahora podemos afrontar el problema de hacer pasar por el subfiltro (I6 b) solanente las secuencias II I- que nos interesan.

İ̀n general las secuencias de clíticos II I- que contienen algún $\mathrm{AC}$. 
(sea o no $C R$ al mismo tiempo) no se ajustan en su orden a la regla (I8) CR-DT-AC:

(I9) Te me presento DT-CR (El CR es al mismo tiempo AC).

(20) Fo te me comia! $A C-C R$

(2I) IEllos te me prescntaron $\rightarrow$ AC-DT (Tú eres el presentado)

D'T-AC (Yo soy el presentado)

(No afirmo, aunque sí lo parezca, que (2r) sea ambigua. Más bien habría que clasificarla como "dudosa»: ante esta oración, los encuestados se dividieron entre las dos interpretaciones AC-DT/DT-AC; otro pequeño grupo la consideró ambigua; en un caso especialniente significativo, una hablante interpretó "ellos te me presentaron» como DT-AC, y uellos te me recomendaron" como AC-DT. Una cosa fue común, sin embargo, en todos los hablantes: el tener que pensarse las respuestas, consecuencia de su falta de seguridad al interpretar las oraciones que se les proponían; lo cual es lógico si aceptamos (I6) ya que, al no pasar por el subfiltro $(I 6 \mathrm{~b})$ tales oraciones, se permite tanto $1 \mathrm{a}$ ambigüedad conıo las dos interpretaciones unívocas opuestas.)

Si llamamos "directa» a toda secuencia de clíticos en la que alguno sea $\mathrm{AC}$ ( $\sin$ tener en cuenta si es, además, $\mathrm{CR}$ ), observamos que son sólo las secuencias II I- "directas» las que permiten todo tipo de violación de la regla (I8) -0 , lo que es lo mismo, las que no deben pasar por el subfiltro ( $I 6 \mathrm{~b}$ ) - E En cambio, si no son directas (si no hay AC entre sus cliticos), deben respetar (I8) para ser gramaticales:

(22) *Te me escapo D'T-CR frente a "Te me escapas" CR-DT.

(23) *Te me como el bocadillo DT-CR

frente a "Te me comes el bocadillo" CR-DT. (Obsérvese que ninguno de los $\mathrm{CR}$ en los dos ejemplos anteriores es a la vez $\mathrm{AC})$.

Podemos generalizar estos hechos retocando el sistema de reglas (I6); que quedaría así:

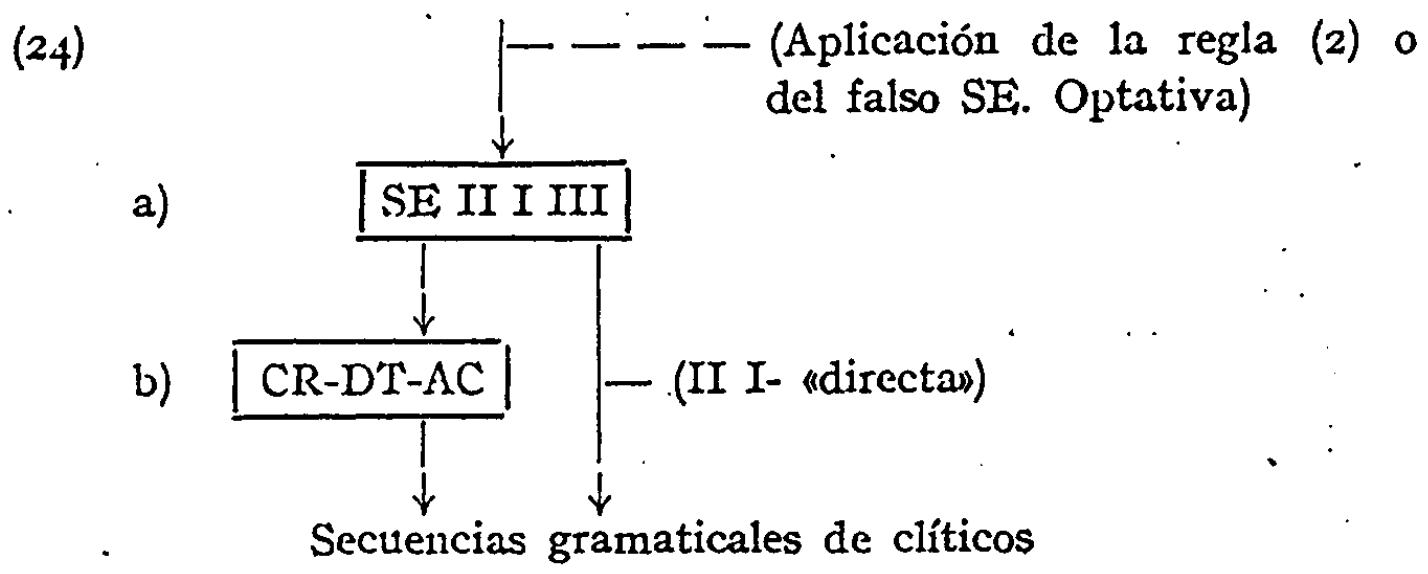


Podcmos entender (24) cono un intento de generalizar la regla tradicional de ordenación de clíticos, para llegar a un conjunto de condiciones necesarias y suficientes de gramaticalidad. Hechos como cl que cl español no permita "*yo te le acerqué» (cntendiendo el movimiento 'ILi $\left.\rightarrow I_{I} I_{i}\right)$ y sí "yo me le acer(qué» (NTE $\rightarrow$ I, Ii), o que "*te me escapé" sea incorrecto frente a "te me escapaste", o que "cllos te me recomendaron" sea ambiguo, reciben explicación colnerente a partir de la regla (24). "Iillos te me recomendaron", por ejemplo, al incorporar una secuencia II I- directa, no pasa por el subfiltro $(24 \mathrm{~b}), y$ permite tanto el orden $\Lambda C-D^{\prime} T$ como $D T-A C$, de ahi su ambigüedad semántica: yo soy el recomendado/tú eres el recomendado. (Pero véase la página anterior. Es intercsante notar que precisamente el grupo de ejemplos anteriores, que escapan de las posibilidades de (I) pero no de las de (24), hacen que Perlmutter escriba que no encuentra explicación sistemática para ellos. En concreto en la nota 25 de la versión francesa, indica que la incorrección de "*yo te le presenté» (AC-DT) no es debida a violación de orden en la secuencia de clíticos; después de (24), sabemos que no cs asfi. In la versión inglesa, comparando "Tí te me escapaste/ * yo te me escapén, apunta que quizá el hecho se deba a una Icstricción noglobal que imponga a determinados verbos el que el clítico reflexivo vaya en primer lugar. Heles Contreras \& J. N. Rojas (r972) recogen y modifican la idea de Perlmutter: en los ejemplos anteriores no nos cncontratíanos ante verdaderos reflexivos, sino ante lo que ellos llaman pseudo-rcflexivos, y precisamente serían éstos sobre los que se aplicaría la restricción no-global: un verdadero reflexivo, como en "te me entregas cn cuerpo y alma", puede no aparecer en primer lugar ("te me entrego...n). Esquematizando la propuesta de Contreras \& Rojas, tendríamos

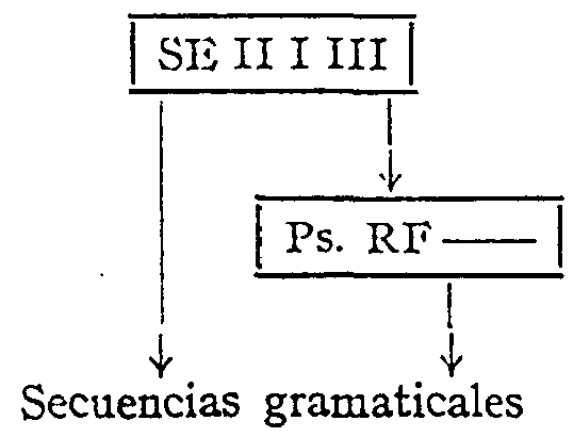

Comparándolo con nuestro esquema (24), hay que notar: primero, no se ha tenido en cuenta que, aparte de la ordenación por personas, bay otra también por categorías (una de las cuales cngloba a los PseudoReflexivos): la incorrección de "*Te me escapé" se debe a la mis- 
ma causa que la de "*me le presentaste», que Perlmutter no se explicaba: el no poder pasar el subfiltro CR-DT-AC. En segundo lugar, Contreras \& Nelson desaprovechan el mayor poder explicativo de su. propuesta: es claro que en "se me escapó» el SE inicial puede ser justificado por el subfiltro inferior, exactamente igual que éste eliminaría "me se escapú" por $n o$ ir el P's. Reflexivo en primer lugar. Ėsto nos liace entrever el carácter redundante del subfiltro inicial al exigir a todo SE. que vaya en cabeza. Sobre todo ello volveremos en $\S$ 4.2.2.)

Por otra parte, tal como está formulada, (24). puede entenderse como filtro colocado a la usalida" del componente transformacional, como conjunto de condiciones eductivas (en el sentido de Perlmutter). Por ello hemos calificado en (24) a la regla del falso SE como optativa: aunque no se aplique, la secuencia IE LO sería marcada como agramatical por (24 a) al implicar III III. La regla del falso SE ha de actuar si se quiere obtener una oración correcta, pero no es técnicamente una. transformación obligatoria, ya que (24 a) la exime de ello.

\section{Algunas culistiones DE teorfa lingüistica geNeral.}

\section{I. Restricciones eductivas vs. restricciones sobre el componente trans- formacional.}

Conviene hacer un alto y evaluar las repercusiones que tendría, en 1a teoria lingüística general, adoptar (24) como mecanismo filtrante de las secuencias de clíticos, colocada a la salida del componente transformacional.

Si partimos del modelo gramatical propuesto en Aspects (la teoria. (standard"), al añadir como mecanismo filtrante una serie de condiciones eductivas, pernitimos que las gramáticas dentro de la nueva tcoría sean enormemente más potentes, y por tanto mucho más numerosas que las derivadas de la teoría anterior. El problema es, en consecuencia, la pérdida de interés de la teoría lingüistica general al hacerse mucho menos restrictiva (alejándose así la posibilidad de un mecanisno. de evaluación de gramáticas descriptivamente adecuadas, 0 , lo que es. lo mismo, la posibilidad de adecuación explicativa). En suma, no podemos propugnar la adopción de restricciones eductivas en una gramática más que si demostramos que, con ello, obtenemos una adecuación descriptiva que de otro modo sería inalcanzable. La pérdida de adecuación explicativa que ello acarrea resultaria así imprescindible, una especie de "mal menor». 
Iin el capítulo segundo (el más importante) de la tesis doctoral de Perlmutter se intenta demostrar la premisa para la adopción de condiciones eductivas por una gramática del español adecuada. Para Perlmutter es entonces tarea fundamental tratar de empequeñecer lo más posible el componente transformacional para compensar la potencia añadicla a la gramática; a cllo dedica el capítulo final de su tesis.

las dos más importantes restricciones sobre el componente transformacional que la adopción de condiciones eductivas permitiría, son las siguicntes:

a) "Blocking Principle»: En el modelo "standard", el componente transformacional absorbe completamente la función de filtro de las cstructuras profundas. Puesto que ahora disponemos de otro mecanismo filtrante, es posible imponer la siguiente condición: "IEl componente transformacional bloquea una derivación solamente en el caso de que una transformación obligatoria no pueda aplicarse porque infringe una metarrestricción de las gramáticass. Es decir, no puede permitirse que sea la aplicación de una $T$. obligatoria la causa del bloquco, sino la inposibilidad de aplicar tal transformación a causa de ciertas restricciones de más alto rango incluidas en la tcoria lingüistica gencral. Por cjemplo, lo que bloquearía una derivación que contuviera una oración de relativo, sin sintagma nominal idéntico al antecedente (cf. Cliomsky, I965, pp. I36-37) no sería una condición ad-hoc sobre la transformación de relativo sino una metacondición general sobre todas las deleciones, que exigiera que éstas no implicaran una pérdida de información irrecuperable.

b) "No-Condition Principle»: Perlmutter, analizando varios ejemplos donde podríanos elegir alternativamente dos soluciones, una que implicara imponer condiciones sobre algunas transformaciones, y otra que utilizara condiciones eductivas, llega a la conclusión de que la segunda es la correcta, Aceptado esto, resulta antieconómico mantener la primera solución en la teoría general, siempre y cuando exista la posibilidad de la segunda: "En las gramáticas de las lenguas naturales no hay condiciones sobre las transformaciones".

Resumiendo, el esquema de la argumentación sería: primero, es incvitable (visto el caso de los clíticos españoles) admitir condiciones eductivas dentro de la teoría lingüistica general; segundo, lo anterior no tiene por qué ir automáticamente en mengua del interés de la teoria, ya que posibilita una serie de importantes restricciones sobre el componente transformacional. 


\section{2. ¿Son inevitables las condiciones eductivas?}

Es claro que la piedra angular de todo el razonamiento de Perlmutter descansa en la demostración de que los fenómenos de orden cu los clíticos del cspañol no pueden ser adecuadamente descritos si no se echa mano de condiciones eductivas. Sin tal demostración nos cncontrainos nada más que ante un modelo sustancialmente distinto del de Aspects, pero sin ninguna ventaja teórica sobre él: restringe el poder del componente transformacional sólo a costa de añadirle un mecanismo sumamente potente a su vez; en este terreno la comparación resulta muy difícil, pero el modelo de Aspects resulta al menos más liomogéneo.

Sin embargo los hechos son muy otros: toda una serie de datos inclinan a pensar que no es posible describir los fenómenos de orden de los clíticos españoles mediante restricciones de la estructura superficial, lo que es exactamente lo contrario de lo afirmado por Perlmutter; en cambio resulta obligado imponer condiciones sobre ciertas transformaciones obligatorias (cn especial la del falso SE) que bloqueen la derivación en casos determinados (compárese esto con los "Blocking" y "No-Condition Principles»). Il demostrar todo ello será el objetivo del parágrafo 4 .

Por este camino llegamos exactamente a la solución opuesta a la de Perlmutter: puesto que en ciertos casos se hace necesario el uso filtrante de las transformaciones sin recurrir a metacondiciones, al elcgir entre una solución de este tipo y otra que conlleve restricciones eductivas, se elegirá sistemáticamente la primera. Siempre en el supuesto de que no haya casos donde resulte imprescindible la segunda; a este respecto es aleccionadora la tesis de Emonds (r970) donde se subraya que todas las restricciones eductivas propuestas por Perlmutter (para el francés, el inglés, el español...) pueden ser formalizadas en una gramática sintagmática.

Cabe señalar también que argumentar que no es lógico permitir a una transformación tan tardía como la del falso SE bloquear toda una derivación (Perlmutter, nota I9 y epílogo) es del todo ineficaz si 110 se señala un mecanismo más general (y menos tardfo) que sea capaz de describir adecuadamente los hechos. En principio, nada hay más tardío ni más ad-hoc que la regla (I) utilizada como testricción cluctiva; y en segundo lugar, tal mecanismo, como veremos, resulta. inadecuado. 
4. LAS RESTRICCIONTSS SOBRE EL ORDEN DE LOS CLÍTICOS NO SON CONDICIONES EDUCTIVAS

\section{I. ¿Puede formularse (24) en el componente transformacional?}

Gran parte de los esfuerzos de Perlmutter van cncaninados a demustrar que (24) -o más bicn (I) - sólo pucde formularse como restricción eductiva. Iffectivamente: podríamos entender (24) como cambio estructural de una transformación de reordenación, o conno conjunto de transformaciones cuyo efecto sea colocar los clíticos en secuencias que cumplau cualquiera de las posibilidades permitidas por (24); en cualquiera de los dos casos llegamos a un callejón sin salida:

- Si colocamos las transformaciones antes de la regla del falso SE, permitimos la secuencia SE SE: una oración como

(25) Miguel se le come el bocadilio a Juan, pero a Domingo no se le lo come

podría ser sin dificultad resultado de una transformación cuyo cambio estructural exigiera condiciones equivalentes a $(24)$; lucgo, al aplicar la regla del falso Sli, obtenctríamos

(26) *Miguel se le come el bocadilio a Juan, pero a Domingo no se se lo come

que la gramática no marcaría como agramatical.

- Si colocamos las transformaciones tras la regla del falso SE, el resultado tampoco es correcto. Sea una estructura profunda del tipo de

(27) Me le como a Juan el bocadillo, pero a Miguel no me le lo como

que (2) transforma en

(28) ... pero a Niguel no me se lo como;

el orden de (28) es claramente incorrecto, pero la aplicación de la transformación reordenadora no arregla las cosas:

(29) *... pero a Miguel no se me lo como sigue siendo incorrecta.

In suma: planteadas así las cosas, la argumentación de Perlmutter cs inatacable, y (24) ha de considerarse como restricción de la estructura superficial. Hay que hacer sin embargo una observación: las dificultades surgen, sistemáticamente, por la aplicación de la regla del falso SE, ya sea antes, ya después de las transformaciones de reordenación. En los contados ejemplos de Perlmutter en que las dificultades provienen de otros clíticos, se debe ello a que él utiliza como cambio estructural el esquema (I) y nosotros el (24), que ya hemos visto es mucho más completo. 
(Es interesante notar que la argumentación de Perlmutter en contra de la formulación de (24) en el componente transformacional alcanza de lleno a un tipo de soluciones de las que Haldlich (r97r) podria ser un ejenplo: se trataría de añadir a las transformaciones de pronominalización y a la del falso SE una serie de transformaciones de reordenación. Lin el caso de Haldlich, especialmente inexacto, las condiciones representadas por ( 24 b) quedan exigidas por el orden (obligatorio) en que se aplican las transformaciones de pronominalización; luego vendría otra transformación equivalente a (24 a). Que tal procedimiento es completamente inadecuado no es difícil de demostrar, remitiéndonos simplemente a los argumentos de Perlmutter antes expuestos, que nos eximen de detenernos en esta cuestión.)

\section{2. ¿Puede formularse (24) como condición eductiva?}

La regla (24), cosa que no habrá escapado al lector atento, está formulada inadecuadamente. Por razones de claridad en la exposición he preferido diferir liasta cste parágrafo el análisis de sus incorrecciones y de los modos de resolverlas, tarea que nos llevará a la conclusión de que las restricciones de clíticos en español no son formulables como condiciones eductivas.

Dos son, en principio, los puntos débiles de (24): su carácter de orden estricto, y la existencia del SE encabezando (24 a).

4.2.I. El problema del orden estricto.

En § 2.I. habíamos observado que (r) -o (24a)- habia que entenderlo como filtro que exigía un orden estricto; ello equivale, como vimos, a añadir a las exigencias puramente de "orden» una serie de condiciones cuantitativas: las secuencias deben tener como máximo un elemento de cada columna; la secucncia II I I no sería permitida, por cjemplo. Salta a la vista que $(24$ b) debe ser retocado si queremos que señale también un orden estricto, ya que puede haber varios $D^{\prime} \mathrm{T}$ en una oración: "ellos me le rompieron la cara al niño». Habría que formular el subfiltro así:

\section{(24 b') CR-DT-DT-AC}

(En la modalidad de español hablada en Galicia habria que permitir incluso tres DT: una oración como "ellos te me le rompieron la cara al niño" no sonaría agramatical a un gallego.) 
Pero ocurrc, además, que el establecer los filtros con la convención de que marquen un oiden estricto, es redundante: como quiera que cntendamos la pronominalización, tenemos que hacerlo de tal modo que tengamos en cucnta ciertas restricciones que imponen los sintagmas nominales correferentes, y entre ellas cstán algunas de carácter cuantitativo. Por cjemplo, hay que especificar en la pronominalización que lus clíticos correferentes con el sujeto no lo sean parcialmente, es decir, exigirles que coincidan en persona y número; con cllo damos cuenta de la gramaticalidad de "yo me cojo el coche" frente a "*yo nos cojo el coche" ${ }^{1}$. De parccida mancra habría que exigir que no hubiera dos clíticos corrcferentes con el mismo sintagma nominal; no es muy correcto decir que esta condición cuantitativa sea una exigcncia del orden de los cliticos: en realidad, ya los introduzcamos transformaciona! mente a partir de una base semántica, ya reciban su lectura semántica (su corrcferencia) tras haber sido incroducidos por la base sintagmática, los clíticos han de cumplir unas condiciones cuantitativas que ya estaban establecidas semánticamente. Podcnos imaginar un significads "yo llevo la maleta a tí, sin esfucrzo» y cxpresarlo mediante la oraciún

\section{$\mathrm{AC} \quad \mathrm{D}^{\prime} \mathrm{T}$}

(30) Yo te $\frac{\text { la }}{D^{\prime} \mathrm{T}} \frac{1 \text { levo } \sin \text { esfuerzo }}{\mathrm{AC}}$

peru como no podemos, en español, pensar a!go así como "yo llevo at tí a tí sin esfuerzo", tampoco podremos realizar tal significado $\overline{\mathrm{AC}} \overline{\mathrm{DT}}$

mediante los clíticos correspondientes:

(3I) *Yo te $\frac{\text { te }}{\mathrm{DT}} \frac{\text { llevo sin esfuerzo }}{\mathrm{AC}}$

$\mathrm{Y}$ no se diga que la oración "yo te llevo a tín contradice la argumminación, porque en ella el pronombre tónico no es sino un refuerzo plconástico

1 Liste hecho indica por sí solo el muy distinto carácter de los clíticos $C R$ del de los $A C$ o D'T. Alarcos Ilorach (1968) considera igual la función (dativo) de MIL; en las dos oracioncs "me como una chuletan y "me comes ma chulcta". Sin enbargo es posible anos comes una chuleta" pero no "nos como...n, ya que en el primer caso tencmos un DT y en el segundo un $\mathrm{CR}$. 
del clítico, y ambos son AC. No se pueden aducir tampoco razones de eufonia: en español una oración como

(32) Nos nos atrevemos a suplicar la paz

cabe dentro del contexto apropiado (en labios del Papa, por ejemplo). Hay que concluir que (3I) es incorrecta porque deriva de una previa violación de carácter semántico.

La condición semántico-cuantitativa (nno puede haber dos clíticos correferenciales") explicaría el que sólo pueda haber un I o II pero sí más de un III: I y II indican correferentes digamos unitarios" y no pueden, por ejemplo, dos ME referirse a personas distintas en la misma oración (y por tanto no pueden coexistir); en cambio en "se lo di" (= "le lo div) tenemos III III cada uno con un referente distinto, debido al carácter no-unitario de la tercera persona.

En suma, al cstablecer (24) como un filtro que exige un orden estricto, expresamos unas condiciones puramente ordinales y otras cuantitativas; las cuantitativas son redundantes. Para subsanar este fallo de la regla podríamos proponerla simplemente como indicadora de un orden: recordemos que, de no ser estricta la relación de orden, posee la propiedad reflexiva; si a la relación uprecede as la llamamos $\mathrm{R}$, será una relación de orden si cumple $\mathrm{xRx}$ ( $\mathrm{x}$ precede a $\mathrm{x}$ ): si CR-DT-AC cs un orden obligatorio no-estricto, una secuencia como CR-DT-DT$A C-A C$ se ajusta a tal orden. Lo que ocurre es que una secuencia así numca llegaría al filtro, ya que sería climinada antes por razones semánticas (bien en el componente semántico interpretativo, bien en la primitiva lectura semántica generadora). La corrección ( 24 b') se hace ahora innecesaria. (cf. § 4.2.I.).

Aceptado que (24) debe entenderse meramente como conjunto de condiciones de orden, ¿cabría entenderlas como condiciones eductivas? No, mientras dejemos como está la regla (2) -del falso SE-. Partiendo de un significado como ise le permite fumar (a usted)", no hay ningún impedimento semántico para llegar a

(33) Se le lo permite

ya que los tres clíticos tienen distinto referente. Ia regla (2) transformaría (33) en

(34) *Se se lo permite y el filtro dejaría pasar tal secuencia ya que no indica un orden estricto. Está claro que si no retocamos la regla (2), capacitándola para eliminar la sccuencia SE-SE, no podemos pretender que (24) sea a la vez una restricción eductiva y una regla no-redundante. 
4.2.2. El problema del SE.

Il segundo punto discutible en la formulación de (24) es el SE. Cuando en (24 a) exigimos el orden SE II I III, reunimos bajo el rótulo SE tanto los clíticos $C R$ de tercera persona (entre los que contamos a los reflexivos, el impersonal, el que forma las oraciones comúnmente conocidas como de "pasiva reflejas...), como el SE originado por la tegla (2), o sea un primitivo LE. De este solapamiento de clíticos pertenecientes a dos categorías distintas (CR y DT) derivan dos importantes defectos que estudiaremos a continuación.

4.2.2.I. La regla (24) es parcialmente redundante, ya que en (24 b) exigimos que todo clítico $C R$ se sitúe en primer lugar, y en $(24$ a) trets cuartos de lo mismo, puesto que gran parte de los cliticos reunidos bajo el rótulo SE son CR. La regla sería igualmente efectiva si elimináramos al SE de (24a), con la condiciún de añadir un mecanismo que obligara a los falsos SE a aparecer en primer lugar. Con cllo, una de las más importantes virtudes de (24) quedaría patente: se trata de una doble ordenación, por una parte relativa a la secucncia obligatoria de "personas" y la otra a la secucncia obligatoria de (funciones):

(35) II I III

$$
\text { CR DT AC - (salvo II I-directa) }
$$

Il extraño postizo que resultaba ser el SE en (24 a) queda asf explicado en parte: salvo los casos de falso SE - que también en esto demuestran su muy especial condición - no hay nada de singular en que el SE impersonal, por ejemplo, haya de situarse en primer lugar siempre, ya que en ello no hace sino comportarse como todo $C R$. Insistimos en que para que (35) resulte con visos de correcta, debe retocarse (2) -regla del falso SE-.

4.2.2.2. Al considerar que todo $\mathrm{SE}$ se ordena de igual mancra en una secuencia de clíticos, estamos describiendo inadecuadamonte el español. En dos casos por lo menos sou diferentes el comportamiento del SI correferencial con el sujeto (CR) y del falso SE (DT); vamos a examinarlos con detalle.

- El SE impersonal (uno de los CR) tiene una curiosa restricción sintagmática: no puede aparecer seguido de LO. Para darse cuenta de ello, no hace falta más que intentar, en una oración impersonal con 
SE, pronominalizar mediante un clítico el complemento directo. En unos casos simplemente no se puede:

(36) Juan dice que... $\rightarrow$ Juan lo dice

(37) Se dice que... $\rightarrow$ * Se lo dice

(37) sería correcta automíticamente si la consideramos personal; "él se lo dicen, en cuyo caso el SE sería D'T.

En otros casos se puede pronominalizar el complemento directo, pero solamente a costa de cometer un leísmo:

(38) Se ejecutó a Servet cruelmente $\longrightarrow$ Se le ejecutó cruelmente *Se lo ejecutó cruelmente Iisto es prácticannente obligatorio incluso para las zonas (y escritores) más reacias al leísmo. Veamos un dato de Fernández Ramírez: los Quintero, cuyo porcentaje de leísmos en oraciones personales es nulo, de doce casos contabilizados de SE (impersonal) + clítico AC III, once las realizan como SE LE (leísmos), y solamente una SE LO:

(39) Se los quiere mucho a estos angelitos.

Iis decir, si el SE es impersonal, cl leismo es obligatorio incluso para los lablantes estrictamente no leístas (para los que el ejemplo (39) es agramatical sin paliativos, del mismo modo, por ejemplo, que tho dí una bofctadan. $Y$ sin embargo éste es un verdadero caso de loísmo, y en el ejemplo de los Quintero el LO debiera ser en principio correcto.)

Lo que es verdaderamente singular en esta restricción es que sólo afecta al LO, ya que la construcción con LA(S) es usada regularmente. Inclusis) se puede detectar una rclativa aceptación en el caso de SE LOS (cf. Alarcos ( $(96)$ ), donde se dan seguidas las dos paráfrasis

$$
\begin{aligned}
& \text { se recibe al embajador } \rightarrow \text { se le recibe } \\
& \text { se recibe a los embajadores } \rightarrow \text { se los recibe). }
\end{aligned}
$$

Si en (39) el complemento clirecto fuera femenino, al pronominalizarlo no es obligatorio el leísmo, sino todo lo contrario:

(40) Se las quiere mucho a estas diablillas ? "Se les quicre mucho a estas diablillas.

Fernández Ranírez contabiliza tres ejeinplos de SE (imp.) LA por uno tan sólo con LE (acusativo femenino): el leísmo no es obligatorio en femenino. (Datos todos ellos tomados de los Quintero.)

Estas extrañas restricciones pueden ser explicadas, con toda la falibilidar del caso, diacrónicamente. Cuervo (p. 95 y ss.) ha descrito así su nacinicnto, en una argumentación que resumo: las impersonales con SE y complemento directo oracional (se mandó que..., se dijo que..., se pensaba hacer...) son anteriores a las que llevan complemento directo 
personal (se niata a los cristianos, se odiaba a los judíos...). Il prinier tipo podía pronominalizar el complemento indirecto (naturalmente, por medio de $L E(S))$ : "se le manda hacer», "se le dice que...", y tal construcción originó que al pronominalizar los complenentos directos de personas, se hiciese - en tcoría incorrectamente- de igual modo mediante LE(S): "Sc les mataba (a los cristianos)", "se les odiaba (a los judíos)". Cucrvo demuestra que, en un principio, tanto los complenentos directos masculinos como los femeninos, los singulares como los plurales, se pronominalizaban en oraciones con SE (imp.) mediante LE(S). La introducción de LA(S) para el femenino sería debida a la presión de1 uso laista en Castilla, y la relativa aceptabilidad del plural LOS para el masculino obedeceria a mimetismo con la construcción francesa cquivalente.

Con ello, se explica perfectamente el que, mientras que el primitivo LE aparezca todavía si es masculino, se cambiara a LA en el femenino: 1a fuerza del laísmo es incomparablemente mayor que la del loísino, que sicmpre se ha considerado de extremada vulgaridad. (En cuanto a la relativa tolerancia de SE LOS, la influcncia galicista que ve Cuervo parcce innecesaria, ya que puede explicarse perfectamente particnclo de la evolución de la estructura de los clíticos de $3 .^{3}$ persona en castellano: como Lapesa ( 1968 ) ha demostrado, el loísmo fue siempre mucho más aceptado -y se inició- en plural.) Sin embargo, la que no aparece muy clara es la razón del total leísmo inicial; la analogía que propone Cucrvo no es razón de peso, por cuanto en las primitivas oraciones tomadas como nodelo (impersonales con complemento directo oracional) tenemos exactamente el mismo problema: en

(4I) Se permite comer a todos los asistentes

podemos pronominalizar el complemento indirecto, pero no se puede hacer lo mismo con el directo:

(42) Se les permite comer

(43) *Se lo permite

En suma, que el quid de la cuestión de la imposibilidad primitiva do utilizar el acusativo de III en una impersonal con SE, creo que se halla cn que tal forma crearía ambigüedad al ser idéntica con la preexistente SE (falso o reflexivo) + acusativo III, que siempre ocurre en oración personal. El resultado actual es que, debido al laísmo, la incompatibilidad sólo recae sobre el LO: "se lo asustó» no se puedc entender sino como "él asustó algo a alguien" (Juan asustó el conejo a Pedro); "se le asusta fácilmente» ha de entenderse como impersonal. De igual manera, "se le lava" hay que entenderlo como impersonal, mientras que "se lo lava" puede entenderse con pron. reflexivo ("él se lava cl cucllo») o con el faso SE 
("él lava el cuello a Juan"), pero siempre en oración personal. Un argumento decisivo en favor de esta tesis (leísmo en oraciones impersonales debido a la concurrencia con la forma (falso SE + LO", siempre personal) lo suministra la gramática de la Academia (1973), que acepta la oración "ruego que sc me lo busque»: si la oración fuera "ruego que se busçue a mi hijo", no podría pronominalizarse con LO, sino "ruego que Se IJE busque»; la mera interferencia de ME en el primer ejemplo, entre SE y LO basta para que la oración ya no se pueda interpretar como creada a partir del falso SE, que sólo aparece inmediatamente antes de $L O(S) / L A(S)$ : el leísmo no tiene ya, por tanto, justificación diacritica y se hace innecesario.

Dejando aparte la anterior discusión, hay un hecho indudable $y$ cs que no podemos agrupar, bajo el rótulo SE, a clíticos tan diferentes en su comportamiento con respecto a las restricciones de orden cono el SE impersonal y el falso SE: el primero no puede aparecer seguido de $I, O$, micntras que el scgundo ha de aplarecer obligatoriamente seguido de $I_{1} O$ si el acusativo es masculino singular (salvo dialectos leístas). Hemos por tanto de recliazar (24), ya que impediria que la gramática diera cuenta de restricciones como la que estamos comentando; esta crítica es pertinente aún con mayor notivo con respecto a (r).

- Hay otro ejemplo claro de la inadecuación de agrupar a todas las ocurrencias superficiales de $\mathrm{SE}$ con respecto a sus restricciones de orden: en una modalidad de español vulgar, es normal anteponer los clfticos de II y I al SE: "me se cayó", "te se marchó la novian (Beinhauer, pp. 47. I49, 222). Thora bicn, este SE es siempre CR, no el falso SE, que mal pucde aparecer con cl orden invertido si jamás ocurre en combinación con ME o TE; es decir, el falso SE se crea, como es notorio si tenemos en cuenta (2), sólo ante $\mathrm{L} O(\mathrm{~S}) / \mathrm{LA}(\mathrm{S})$, y por tanto la particularidad del ME SE/TE SE no le afecta.

$\mathrm{Si}$ intentamos describir las restricciones de orden de los clíticos en tal modalidad del español, no nos basta con retocar (24), admitiendo también el orden II I SE III, por ejemplo, sino que hemos de especificar de qué SE se trata. En Arniches encontramos (citado por Fernández Ramirez):

(44) Habia ido el gato y me se lo habla comido que sería perfectamente aceptable en el habla achulada madrileña, pero lo que jamás se consideraria gramatical sería transformar nyo me le comí su comidas en

(45) Yo me le la comi = *Yo me se la comí MIE SE LO sólo pucde admitirse si el SE es CR, nunca si es el falso SE. El becho tiene su importancia también para el español normativo (cul- 
to): en "me se acercó" percibimos una violación de orden, pero ante "yo me se acerqué» 110 pensamos en ello, sino en un fallo de fonación o de audición, ya que el orden inverso tampoco sería correcto; son dos oraciones agramaticales pero no del mismo modo, exactamente igual que (44) y (45). Atribtyendo - como hace la regla (24)- la incorreción de ambas parejas a una violación de orden, no representamos adecuadamente la competencia del hablante.

\subsubsection{Primeras conclinsiones.}

Hemos visto en $\S 4.2 .1$. que (24) es redundante al marcar unas restricciones cuantitativas que ya hay que tener en cuenta en el mecanismo de la pronominalización. La conclusión es que (24) debe entenderse conno restricción de orden puramente, lo cual implica que si debemos seguir entendiéndolo cono conjunto de condiciones eductivas, tenemos que modificar (2) - regla del falso SE- para que bloquee la derivación, si de su aplicación resulta la secuencia SE SE.

Vimos lucgo, en $\S$ 4.2.2.I., cómo es también redundante indicar que SIi (CR) vaya en primer lugar, ya que en eso se comporta como todo CR. Indicamos además $-\$ 4 \cdot 2.2 .2$. - que no es correcto agrupar a todas las ocurrencias de SE indiscriminadamente, en relación con sus restricciones de orden, ya que en este sentido se comportan de muy distinta manera los CR y los falsos SE. A la vista de todas estas deficiencias, es evidente que se impone una formulación del tipo (35), esto es, haciendo desaparecer al polivalente SI:

(35) II I III

CR D'T AC (salvo II I- directas)

solución que lleva aparejado el modificar la regla (2) de modo que no sólo indique que IE $+\mathrm{AC} I I I=\mathrm{SE}+\mathrm{AC}$ III, sino que además prohíba que tal transformación ocurra en cualquier otro tipo de secuencia.

Contreras \& Nelson (I972) llegan a conclusiones parcialmente coincidentes: aceptan la validez de (I) cono condición eductiva en lo que se refiere a II I III, pero no en cuanto a SE, que debe ser tratado transformacionalmente. Su argumentación, a grandes rasgos, se basa en la agramaticalidad de "*se puede bañarse», frente a "se prohíbe bañarse». Esta disparidad se debería a que la primera es una oración simple, mientras que la segunda sería compleja, pero esto no pueden tenerlo en cuenta las condiciones eductivas tal como Perlmutter las expone. Naturalmente podría proponerse otra condición de este tipo que indicara que no pucde haber más de un SIi en una oración simple, pero - como indican Contreras \& Nelson- cllo atribuiría a causas distintas la agrama- 
ticalidad de "*se se baña» y "*se puede bañarse», que básicamente cs la misma. La solución para estos autores es colocar posteriormente a todas las transformaciones de reflexivización la transformación que sustituye un sujeto indeterminado por un SE impersonal, con la condición de que no haya en la misma oración (simple) otro SE. La objeción es en principio acertada, pero tiene los siguientes inconvenientes:

Primero, no es necesario salirse del marco de las condiciones eductivas para resolver la dificultad indicada: lo único que habría que añadirle a éstas es el ser usensibles» a la estructura oracional a la que se aplican, impidiendo que lo hagan oraciones complejas (naturalmente, habria que retocar a fondo la formulación de (I)): esta solución la señala M. Rivero (I973) (es una recensión de Perlmutter ( 1968 ), para superar oraciones del tipo "el hombre que quería lavarse se fue», que Einonds (r970) proponía en contra de ( $\mathrm{r})$ ).

En segundo lugar, la dificultad no sólo surge con el SE. Igualmenteencontramos

Te prolíbo lamentarte vs. ${ }^{*}$ Me puedo lavarme
Me olvidé de lavarme

Habria, por tanto, que impedir la introducción de todo reflexivo en una. oración simple, siempre que ya existiera otro igual; y no sólo todo reflexivo sino todo clítico:

El hombre que lo hizo, lo rompió vs. *El hombre lo puede hacerlo.

Pero si cutendemos estas restricciones cuantitativas como restricciones scmánticas, cosa por otra parte difícilmente evitable, el problema recibe una solución natural e inmediata: en una oración simple no puede haber dos clíticos con el mismo correferente. En "*se puede lavarsen, por ejemplo, ambos SE se originan a partir de un mismo sujeto profundo (cf. Contreras \& Nelson, y Schroten para más detalles), y por tanto son incompatibles ya que se trata de una oración simple. La restricción semántica elininina además automáticamente oracionesdel tipo "*ine puedo lavarnos", cosa fuera del alcance tanto de la solución de Perlinutter-Rivero, como de la de Contreras \& Nelson (a no ser que introduzcamos todo un complejo aparato de condiciones sobre la reflexivización).

En suma: para solucionar los problemas planteados a (24) por el carácter estricto del orden, debemos modificar la regla (2). Para solucionar los problemas planteados por el SE, debemos igualmente retocar la regla (2). $A$ estas alturas no resultará una sorpresa que tales modificaciones sean una y la misma: para posibilitar que (35) marque las restric- 
ciones de orden no-estricto de los clíticos, basta con que antes se aplique

$$
\text { I }-\left[\begin{array}{c}
\text { Pro } \\
\text { III } \\
\mathrm{DT} \\
2
\end{array}\right]\left[\begin{array}{c}
\text { Pro } \\
\mathrm{III} \\
\mathrm{AC} \\
3
\end{array}\right] \underset{\mathrm{I}+\mathrm{SI}+3 \text { (si I no contiene }}{\text { clíticos) }} \begin{gathered}
\text { Bloqueo (si I contiene al- } \\
\text { gún clítico) }
\end{gathered}
$$

Ia regla (46) sólo crea el falso SE si con ello da lugar a la secuencia aislada $\mathrm{SI}+\mathrm{LO}(\mathrm{S}) / \mathrm{LA}(\mathrm{S})$. Entre las cosas que ello implica, naturalniente, está el que el faso SE aparezca obligatoriamente en primer lugar.

Aceptada la modificación (46) ipodría entenderse (35) como restricción de la estructura superficial? Evidentemente no. Vimos en $\S 3.2$. que solamente podriamos aceptar el introducir condiciones eductivas en una gramática en dos supuestos: a) que ello sea inevitable si queremos que la gramática sea válida; b) que ello suponga una alternativa correcta, y más simple que otra también correcta. En cuanto a lo primero, sabemos por § 4.I. que lo único que impedia el admitir una transformación de reordenación de clíticos era la formulación de la regla del falso SE como (2), que permitía la creación del SE incluso con clíticos precedentes. Al transformar (2) en (46), tal problema desaparece. Lo que es más importante: (46) es indispensable si queremos que la gramática sea descriptivamente adecuada.

Nos queda la segunda posibilidad: si el considerar (35) como condición cductiva simplificara la gramática, deberianos aceptarla como tal. Pero no es así, ya que, al tener que rechazar (2) en favor de (46), admitimos que el componente transformacional debe tener el poder de bloquear una derivación ( $y$ actuar por tanto como filtro) sin tener que ceñirse a metacondiciones: o, lo que es lo mismo, admitimos que no es posible aceptar el Blocking Principle ni el No-Condition Principle (cf. $\S$ 3.I.), ni por tanto simplificar el componente transformacional en favor de un aparato de restricciones superficiales.

Resulta, entonces, que el proponer (35) como condición eductiva es una alternativa más complicada que el entenderlo como transformación reordenadora de clíticos, ya que en este segundo caso asignamos un gran poder filtrante al componente transformacional, pero en el primero añadimos a tal poder el de las condiciones eductivas. La conclusión lógica es que (35) no puede entenderse como tal condición eductiva. 
5. IEsquema de una solución. Conclustones

El conjunto de restricciones sobre la secuencia de clíticos que (24) representa, es en realidad una amalgama de restricciones de muy diversa clase. Por una parte cuantitativas, formulables en el mecanismo de la pronominalización. Por otra, restricciones puramente de orden en cl componente transformacional. Finalmente, las restricciones más ideosincráticas, casi morfonológicas: las que regulan la aparición del falso SE, la imposibilidad de la secuencia SE (imp.) LO, etc. El dar una posible formulación a este heterogéneo conjunto no puede circunscribirse, ya lo hemos visto, a reunir en una fórmula concisa hechos muy dispares: hay que aceptar que la verdadera solución es más complicada, quizá menos brillante $y$, desde luego, nada sencilla. Intentaremos ahora dar sus grandes líneas.

Partamos de un modelo gramatical como el propuesto por Jackendoff (I93I), cap. I, por ejemplo. (No entramos alora en la posible discusión de tal elección; simplemente quiero señalar cómo podrian formularse las restricciones en un modelo gramatical suficiente.) Las reglas sintagmáticas de la base introducen todas las formas cliticas y les asignan una función, exactamente igual que a cualquier sintagma nominal. Los rasgos particulares de cada forma (persona a que pertenecen, el ser forma especializada en $\mathrm{CR}$ - como el $\mathrm{SE}$ - ...) están indicados en el Lexicón. En el componente semántico cada clítico recibe una lectura referencial adecuada a sus rasgos particulares y función: un (AC III), por ejemplo, quedará marcado como correferencial del sintagma nominal complemento directo (pudiendo además ser correferencial con el sujeto). Tendríamos entonces las diferentes categorías de clíticos que indicábamos en $\S 2.3 .4 .:$

- CR: correferenciales con el sintagma nominal sujeto. (Podemos distinguir además el tipo IMP., que es sujeto.) Cualquier forma puede ser CR pero el SE es obligatorio para la tercera persona.

- AC: correferenciales con el sintagma nominal complemento directo. Todas las formas pueden serlo, pero en tercera persona son obligatorios $\mathrm{L}, \mathrm{O}(\mathrm{S}) / \mathrm{LA}(\mathrm{S})$ salvo modalidades leístas.

- DT: correferenciales con un complemento distinto del directo. En tercera persona solamente LI(S), salvo loísmos o laísmos.

(Las tres categorfas no son excluyentes: un $C R$ puede ser, al mismo tiempo, AC o DT). 
Ein cuanto a las personas, tenemos:

- I: MUi/NOS

- II: TE/OS

- III: LO(S)/LA(S)/LE(S)

Podemos formular ya los tres grandes grupos de restricciones:

A.-Componente SEMÁNTico: restricciones cuantitativas.

Hay varias condiciones semánticas que debe cumplir un clítico a la hora de asignársele una lectura referencial:

I) Un clítico no puede ser parcialnente correferencial con un sintagma nominal (o sea, han de coincidir en persona y número): ello asigna una lectura semántica anormal a la estructura profunda ryo nos como un bocadillo".

2) No pucde haber dos clíticos correferenciales: lo que marca como semánticamente anormales estructuras profundas como "yo me nos como el bocadillo", $y$ "él se se pasea".

B.-COMPONENTE TRANSFORMTACIONAL.

I) Restricciones de orden: Toda secuencia de clíticos deberá sufrir una transformación obligatoria que la reorganice de tal modo que cumpla dos restricciones, una relativa al orden de las personas y la otra al de las categorias:

$\left[\begin{array}{l}\text { Conjunto } \mathrm{X} \text { de } \\ \text { Clíticos en orden I }\end{array}\right] \longrightarrow\left[\begin{array}{l}\text { Conjunto X de } \\ \text { Clíticos en orden 2 }\end{array}\right]$

2 debe cumplir simultáneamente:

a) II I III

b) CR DT AC (salvo II I- directa). Cf. 2.3.4.

Obsérvese que hay secuencias que no pueden cumplir a la vez a) y b), y por tanto bloquearán la derivación, quedando marcadas como agramaticales por ordenamiento incorrecto: "*él me le acercón (ME $\rightarrow$ LE).

Las dificultades que aparccían con las secuencias MIE LE/TE LE y II I-, aparecen resucltas con este doble ordenamiento. (Cf. $\S 2.3 .1 ., 2.3 .3$.$) .$ 
2) Restriccioncs particulares:

- - - Restricciones del falso SE:

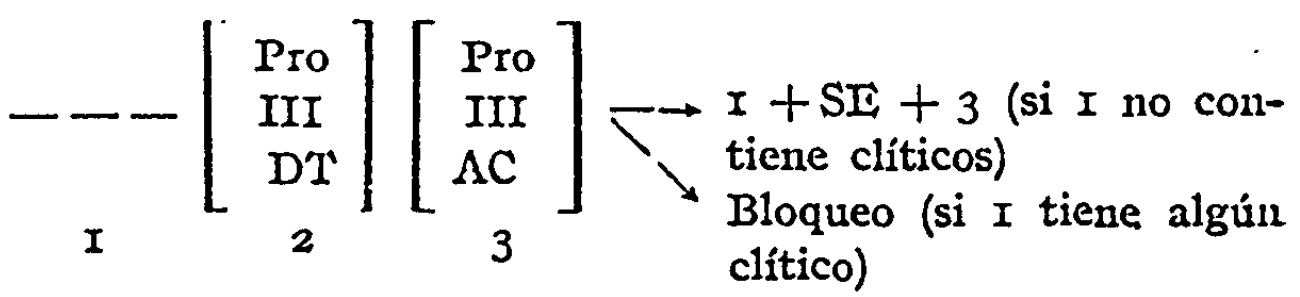

Oraciones como "yo me le lo cogi" (provinientes, por ejemplo, de nyo me le cogí el coche), no están marcadas hasta ahora como agramaticales ni semánticamente (obsérvese que cumple Ar y A2), ni por violar la regla de orden (BI). Esa es la razón de que "*yo me se lo cogi" no se vuelva gramatical al pasar el SE al primer lugar, ya que su orden no era incorrecto (justo lo contrario de "une sc cayó», por ejemplo). Es la regla del falso SE la que marca su agramaticalidad, ya que el LE 110 puede pasar a SE al haber un clítico anterior.

\section{_ _ - Restricción del SI impersonal:}

Uno de los pronombres $C R$ es el IMP. Vimos que no podía aparecer en la secuencia SE LO. Hay casos donde no cabe pronominalización del complemento directo (se dice que... $\rightarrow$ *se lo dice) que podríamos generalizar provisionalmente como koraciones impersonales con complemento directo oracionali. En otros casos es obligatorio el leísmo al pronominalizar el conplemento directo. Podríamos formular todo esto asf:

$$
\begin{aligned}
& {[\text { IMPP }]+[\mathrm{LO}] \longrightarrow \text { I + LE (si } 2 \text { es correferencial con sintagma }} \\
& \text { I } \\
& 2 \\
& \text { nominal no-oracional) } \\
& \text { Bloqueo (si } 2 \text { es correferencial con sintagma } \\
& \text { nominal oracional). }
\end{aligned}
$$

(Cf. § 4.2.2.2.). De este modo "se asusta fácilmente a Juan" se generaría incialmente - con clíticos- como "se lo asusta fácilmente", pasando así al componente semántico, pero transformándose mediante la regla que acabamos de formular en "se le asusta fácilmente», que es como llegaria finalmente al componente fonológico.

Con A ( I-2) y B (I-2) integramos en la gramática todo el conjunto de restricciones sobre las secuencias de clíticos que no dependen del ver- 
bo de la oración: obsérvese que cll ninguna de las reglas se menciona a éste en absoluto. Por cllo, no explican el por qué es correcto "me escapo" y no lo es "*lo escapo", aunque sí nos informan de que tal incorrección no obedcce a restricciones sobre los clíticos (lo contrario de "*yo nos escapo", que AI marca conno agramatical). En cuanto al conjunto de condiciones suficientes y neccsarias sobre el orden de las secuencias de clíticos, está formulado en BI, como transformación obligatoria.

En resumen, la agramaticalidad de las oraciones

(47) *Te os acercaste vs. Te nos acercaste

(48) *Me se vino encima vs. Se me vino encima

(49) *Yo me se lo comí vs. *El me se lo comió

(50) *A Oswald no se lo llegó a juzgar

se origina en muy diferentes partes de la gramática, y no se puede echarlas en el cajón de sastre que la regla (I) representa: (47) viola reglas de naturalcza scmántica, (48) infringe el orden obligatorio de los clíticos, (49) y (50) se debcn a particularidades del falso SIì y del SE impersonal respectivancnte.

Finalmente, nótese que el conjunto $A+B$ es válido tanto para los dialectos que respetan etimológicamente los clíticos, como para los leístas y laístas.

Madrid, C. S. I. C.

\section{SALVADOR BASTIDA}

BIBIIOGRAII $\Lambda$

A cavimu Iispañora, Gramática de la lengua española. Miadrid, İ̀spasa-Calpe, 1931.

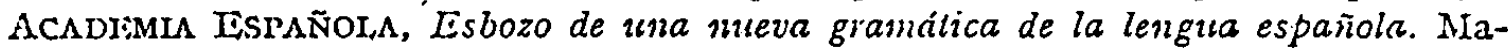
drid, Iispasa-Calpe, I 973 .

ALARCOS Li,ORACH, E., Valores de/SE/. Archivzm, 1968, XVIII. Recogido en Estudios de gramálica funcional del español. Madrid, Gredos, I971, pp. I56-165.

ALONSO, A. \& IHeNRfQUEZ UREÑA, P., Gramática castellana, I7.8 ed. Buenos Aires, Iosada, I96r.

BACח, E., Nouns and Noun Phrases. En Bach \& Harnus (eds.), Universals in Linguistic Theory. N. York, Holt, Rinehart \& Winston, I968, pp. 9I-I 24.

benNenutir, W., El español coloquial. Madrid, Gredos, ig68 (2.8 ed.).

BriLr.o, A., Gramática de la lengua castellana. 8. ${ }^{2}$ cd. Bucnos Aircs, Sopena, I970. CArbali, Carisro, R., Gramática elcmental del gallego cominn. Vigo, Galaxia, r 966. ConTrusiras, II., Grammalicalily us. Acceptability: The Spanish SE Case. Linguistic Inquiry, 1973, pp. 83-88. 
Contreras, H. \& J. N. Rojas., Some Remarcs on Spanish Clitics. Linguistic Irquiry, I972, pp. 383-392.

Corervo, R. J., Los casos encliticos y procliticos del pronombre de tercera persona. en castellano, II. Romania, 1895, XXIV, pp. 219-263 (Apéndice I, En frases impersonaless, pp. 245-252).

Cromsky, N., Aspects of the Theory of Syntax. Cambridge (Mass.), MITI Press, 1965. Cito por la traducción española. Madrid, Aguilar, 1970.

EMonds, J. E., Root and Structure-Preserving Transformations. Tesis Doctoral en el MIT. Publicada a multicopista por el Ininguistic Club of Indiana University (Bloomington, Indiana), 1970.

FERNANDEZ RAMfRez, S., Gramática española. Los sonidos, el nombre, el pronombre. Madrid, Revista de Occidente, I95I.

GruI y GayA, S., Curso superior de sintaxis española. Barcelona, Spes, 196r (8. ed.).

IIAInIXCr, R. I., A Tramsformational Grammar of Spanish. Inglewood Cliffs. (N. J.), Prentice-Hall, 197x. Cito por la traducción española. Madrid,. Gredos, 1973.

HIIGER, K., La conjugaison objetive en francais et en espagnol. Langages, 1966, III, Pp. 18-39.

Hur,ron, J. S., Placcment of Objcct.Pronouns in Spanish. IIispantia, x960, XIIII, pp. $584-5$.

JACKI:NDOFr, R. S., Semantic Interpretation in Generative Grammar. Cambrigde (MIass.), MITT Press, I972.

IAAPES, R., Sobre los origenes y evolución del letsmo, latsmo y loismo. In Festschrift W. v. Wartburg. Tubinga, Max Niemeyer, 1968, pp. 523-5 I.

IICCANLEy. J. D., Where Do Nontr-Phrases Come from? En Jacobs \& Rosenbaum (eds.), Readings in English Transformational Grammar. Waltham (Mass.), Ginn \& Co., 1970, pp. 162-183.

BIOEN, MLARY E., The Fable of Malapropish Affixes. Modern Language Journal, I966, I, pp. 8-II.

OCA, E., El pronombre SE en nominativo, BRAE, 1914, pp. 573-58r.

PERLMUTTIR, D., Deep and Surface Structure Constraints in Symtax. Is su Tesis Doctoral (MIIT, r968). Publicada en N. York, FIolt, Rinehart \& Winston, 1971. Del capitulo 2.0 (referente a los cliticos del español! se ha publicado una versión francesa muy modificada: Langages, r969, XIV, pp. 81-r33. Salvo advertencia en contrario, cito por la edición inglesa. [En Linguistic Inquiry, 1970, I, pp. I87-255, ha aparecido también el cap. $20^{\circ}$ en la versión original inglesa.]

RIVRo, MI., Recensión de Perlmutter, r968. Langiage, 1973, pp. 697-701.

ScIrroten, J., Concerning the Deep Structures of Spanish Reflexive Sentences. La Haya, Mlouton, 1972.

No he podido consultar E. V. THOMSEN, The Generation and Surface Ordering of Spantish Clitics, Tesis Doctoral (Texas, 1969) inédita. De cualquier forma, Haldlich (p. 132, n. 59) elabora su capitulo sobre los clíticos del español basándose en esta obra. 